\title{
The Application of Chinese Traditional Literati Painting in Animation Design
}

\author{
Zhe Ji \\ Xiamen University of Technology, Xiamen, Fujian, China, 361024
}

Keywords: traditional literati painting, animation design, modeling, space scene, composition layout

\begin{abstract}
As a member of the art family, animation has many creative commonalities with other artistic forms in terms of creative thinking and methods. The author learns from these rich artistic forms in modern animation short film creation language, and explores the breakthrough of animation short film creation language. Based on the author's learning and practical experience, this paper analyzes the representation of Chinese traditional literati painting in modeling, the application of space scene, the expression of color, and the expression of composition layout. A thorough understanding for the characteristics of literati paintings creations and modern animated short films is helpful to apply the artistic characteristics and artistic methods of literati paintings in modern animation short film creation, which can make modern animation short film creation language have new expression in the form and content.
\end{abstract}

\section{Introduction}

Literati painting, as a quintessence of world art and culture, has its own unique charm both in artistic expression and in creative expression, and it is a model for artistic creators to learn. Modern animation creation cannot blindly pursue and copy the original animation expression, and create works should not be like fast food culture with little nutrition. Animation works should inherit and carry forward the characteristics of the cultures of all nationalities in China, and should pay more attention to the expression of emotional thoughts and creative language, rather than relying on modern animation technology to show the numerous pictures and exciting visual effects. Animation design should inherit and develop traditional Chinese humanistic paintings to create more distinctive works.

\section{The Embodiment on the Modeling}

\subsection{Line and Structure}

The lines drawn by the literati give people a sense of ease. Careful observation of the seemingly casual pen shows the author's confidence in observing the painting and in knowing the structure. Only when you have a thorough understanding of what you draw, will each thread be rooted in place and drawn to the right place. In Huang Shen's picture of Zhang Guolao riding a donkey (Fig 1), the old man raised his head to the upper right, raised his legs, and rode leisurely on the donkey. The lines of the head are accurately delineated, especially the depiction of the eyes, vividly showing the contentment of the aged. The lines of the beard and the lines of the loose clothes are in sharp contrast with the brush method, which well express the texture and internal structure of different things ${ }^{[1]}$. Especially, the expression of the pleat, each line between the sparse and dense is under the hypertrophic clothes. We can see that the literati painters' seemingly random writing is based on a deep understanding of things. The creation of modern animation in modeling is also inseparable from the lines and structures, especially when we create two-dimensional animation, the requirements for lines are very harsh. Because the lines in different two-dimensional animation are not the same as those in literati painting, it requires the line flow and the fusion of the whole picture. 


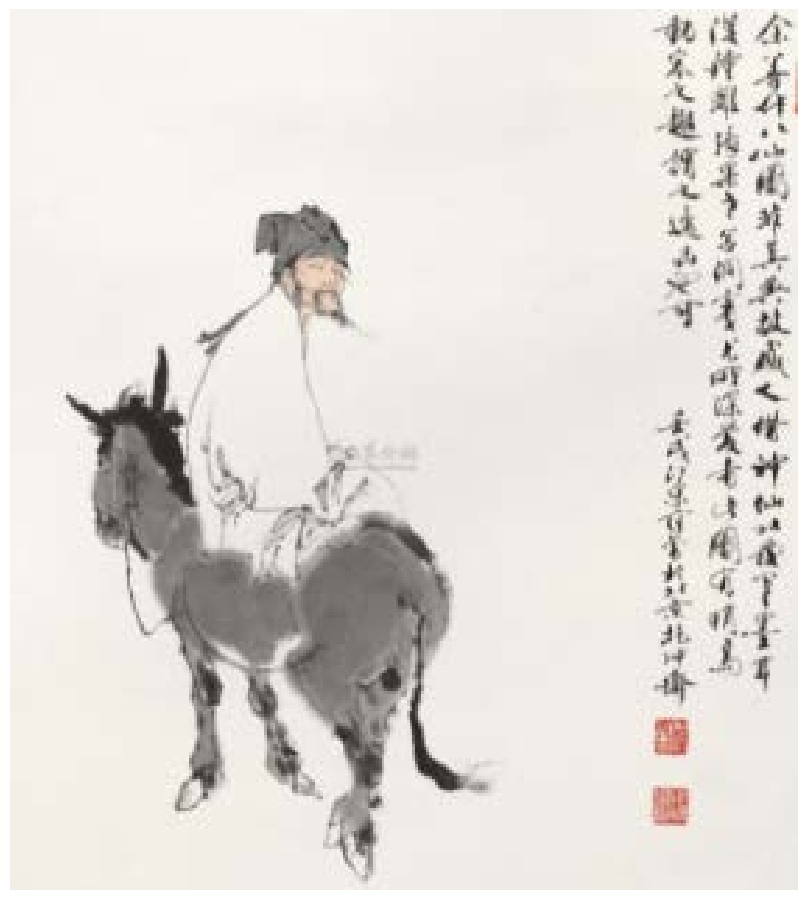

Fig 1. Zhang Guolao riding a donkey.

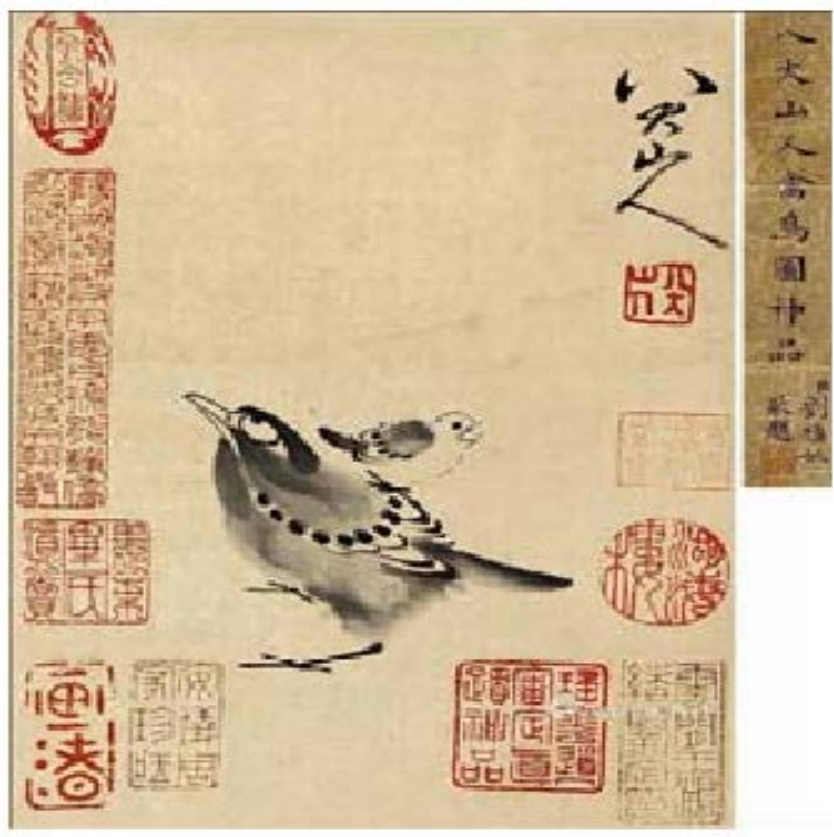

Fig 2. Bird

\subsection{Simplicity and Complexity of Modelling}

When modern animation creates images, sometimes it makes the shape full too complex design to resonate with the audience and to attract attention. In order to simplify, some animation modeling lost its characteristics, there was a problem of inaccurate pinching. Although the literati painting in the form seems simple, it has strong generality. The author thinks that this is the realm that modern animation pursues in modeling design. Through the bird (Fig 2) of Badashanren, we can see that the bird steps on the back of the big bird and sings clearly. The performance of the two birds in the same painting is very different, the big birds are stable, the wing details are clear, and the birds on the back are in the position of singing. But in the overall, the performance of the wings was simplified. Two birds are a clear combination of simplicity and complexity. Bird simplification not only does not weaken its image, but also highlights its lightness, living waves, which makes it seem to hear its crisp sound ${ }^{[2]}$. 


\subsection{Concrete and Abstract}

Literati paintings show the characteristics of things by concrete, and sometimes convey emotion and artistic conception through abstraction to show the inner charm of things. Literati painting, according to the need of creation, should change different forms of expression to express what they think. Concrete and abstract idea both exist together, and the author thinks abstract idea comes from concrete idea. In creation, there are subjective things as well as the influence of objective things. Abstract ideas are often the subjective reflection of concrete objective things. Li Keran's Picture of Children and Old Men (Fig 3) is a work used abstract and figurative idea properly. It not only has the concrete sense of modern sketches, but also has the abstract taste of the literati with splash-ink brushwork, and it is also a rare work of art fusion between China and the West. Nowadays, animation is influenced by western art, and the creators' method thinking is too rigid and lacks the unique features of abstract form in the literati paintings in order to make everything concrete and pursue the so-called extreme ${ }^{[3]}$.

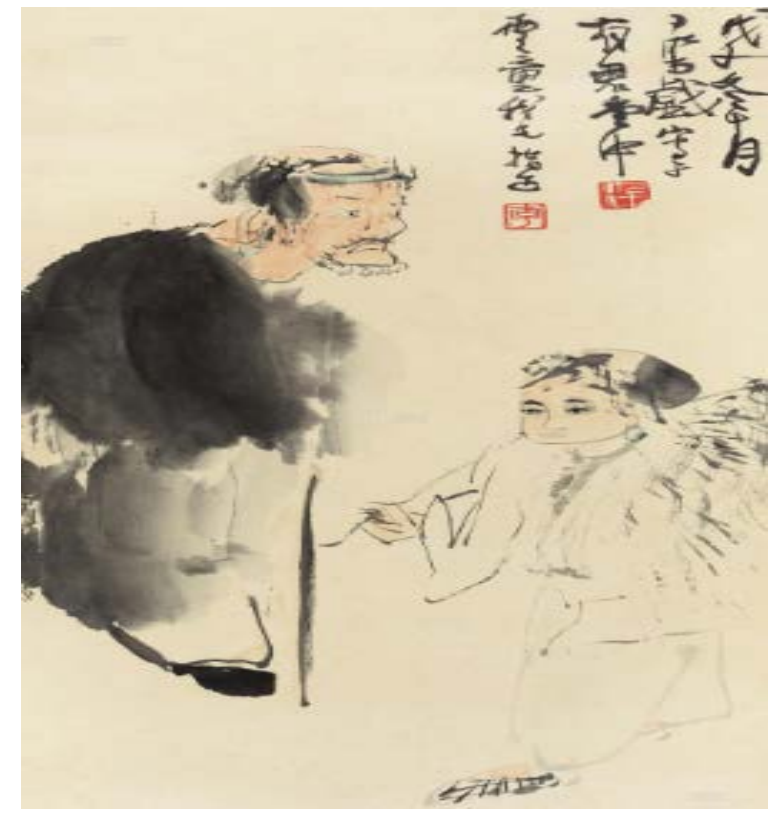

Fig 3. Children and Old Men.

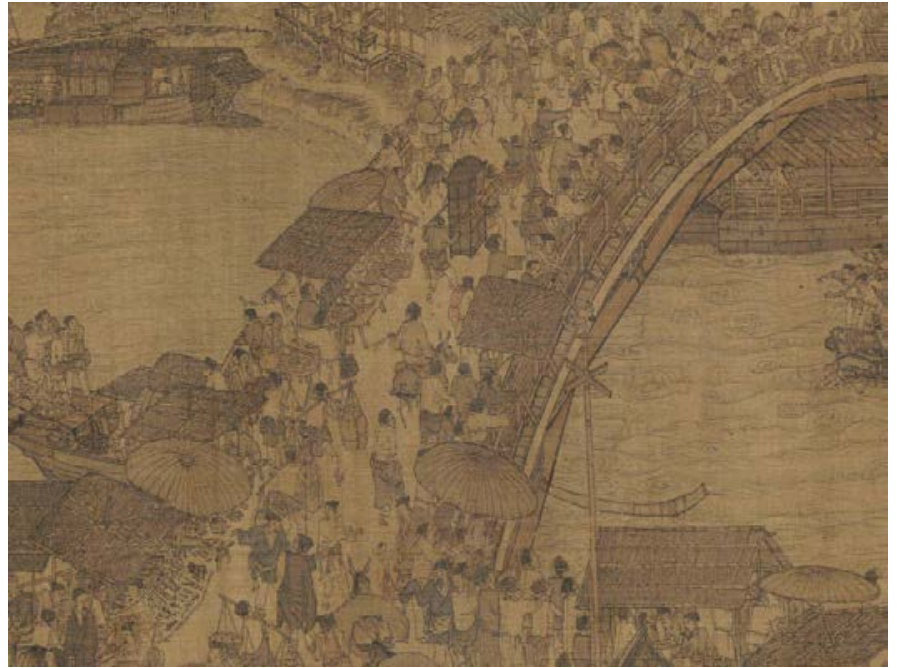

Fig 4. Riverside Scene at Qingming Festival.

\section{The Application of Spatial Scene Representation}

Expression of spatial perspective. There are different ways to express space in different art forms. The scattered point perspective in the paintings of the literati is more to create a free space. The 
scattered point perspective has a natural advantage in the presentation of the narrative and the large space impression of the whole space, and the point perspective has a unique style of decoration. Compared with the focus perspective of the West, we are more receptive to their definition of space, to the modern animation in the spatial expression of the focus perspective, one point, two points, three points perspective. We observe the characteristics of different spatial expression techniques through works, for example, the Northern Song Dynasty Zhang Zeduan's Riverside Scene at Qingming Festival (fig.4). The picture depicts the suburban scenery - the houses are low, the fields crisscross, the people come and go, the overall contents of the picture are colorful and full, and the characters are vivid, which shows the real life appearance of the prosperity of the capital city in Northern Song Dynasty. The carriages go back and forth on the bridge, pedestrians come and go, and commerce is intense ${ }^{[4]}$. A ship to put down the mast is going through a low bridge, the boatmen move carefully, and many pedestrians stood by, these are depicted as lifelike. The picture is presented by scattered point perspective, which uses the moving characteristics of human eyes, showing broad horizon, coherent space and the characteristics of scene moving with people.

Vacuity and reality. Near real far deficiency is often our basic understanding of the void and reality of space. In order to make the viewer focus on the main part, the virtual and real in the literati painting have the function to express the dynamic feeling and the artistic conception. Virtual and real do not blindly refer to ambiguity and clarity, sometimes the performance of the virtual reality is based on the characteristics of different objects in the scene. The draught fog and the stream flowing down the mountain stream in literati's paintings are all based on the physical characteristics of things in reality. In modern animation, the virtual and real expression of space is often expressed by the method of camera focus focusing, which is too common. In the creation of animation scene space, we can use literati painting for reference to try and innovate the expression mode of different things' own characteristics. In fact, we can also see the application of this way in the earlier animated short films. In the reed pipe (Fig 5), the mountains and waterfalls income are decimated. The virtual performance of mountains is the distance of space, and the virtual expression of water is the activity of water. The shepherd boy and his cattle are embodied in reality, and when the cattle are launched, the outline of the cattle is empty to show the fluidity of the water. We can see that the virtual and real scenes can be changed with different emotions and different environments, which is also the reference for future animators.

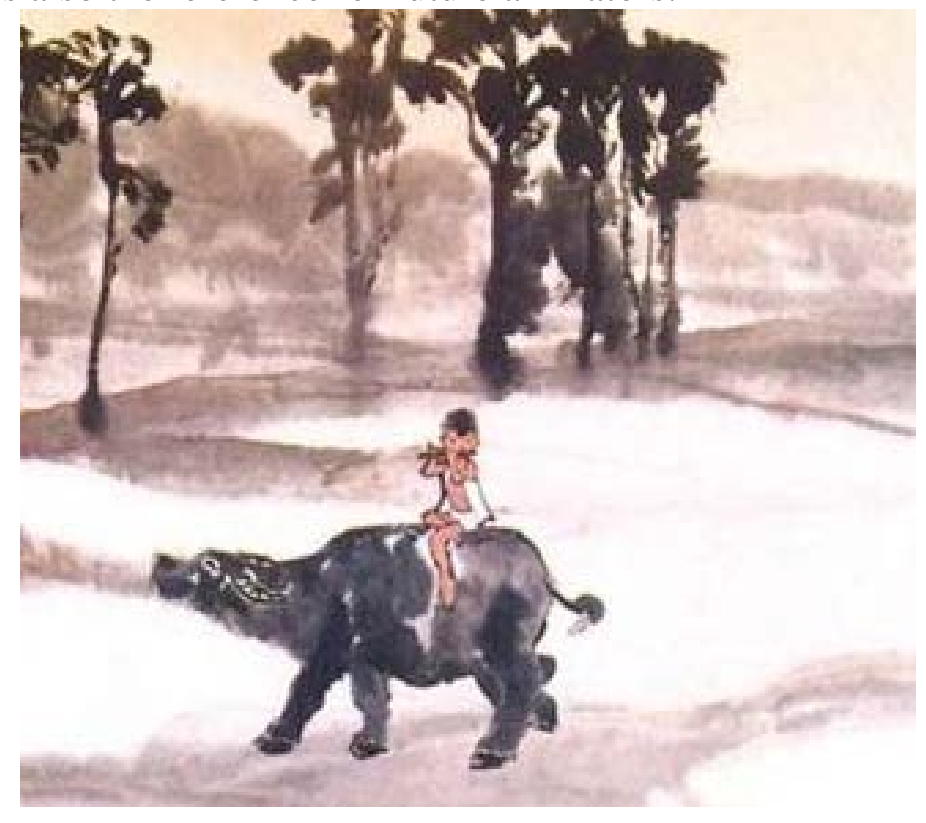

Fig 5. Reed Pipe

\section{Performance in Color}

For color, the literati painting of five colors - thick, light, dry, wet, black are well known for us, 
and the application of ink five colors can be described as the unique performance of literati painting. Literati painters use the physical characteristics of dry and wet ink to express the subtle changes in their works and to express the colorful world with pure ink. This kind of ink-color expression technique seems to be single, but the expression of the world is changing thousands of times. In addition to the five-color ink, literati painting also painted to paint things to express green mountains and rivers and other things. For example, in the Ming Dynasty, Qiu Ying was good at white painting, ink painting, and color setting. He also had a unique understanding of ink painting and light color landscape creation, but in the great green landscape painting, he showed the expression of the heavy color. His painting were praised by colleagues at that time and by modern generations ${ }^{[5]}$. When he was painting the rocks, he smoked them with light ochre, and then, according to the physical characteristics of the stone surface and the image in the natural light, he dyed again with dark green and ink blue. In the creation of animated short films, the color is more intuitive to express the theme of the work, from the side to reflect the personal feelings and ideological tendencies of the creator, has a special symbolic significance. In addition to using language movements to express the character's inner emotional and psychological state, it also uses color to carry on the hint. In the Lion King, in order to show Uncle Simba's evil, a large number of cool colors are used in color design to imply that these methods of color use seem to have become a symbol. However, the expression color intermingling and permeating is relatively rare in the creation of modern animated short films. It is difficult to do this in many modern animations in the use of color. Sometimes, they just paint the color mechanical without caring about the inner changes of the character, so that the work is lifeless.

\section{Representation in Composition Layout}

Although literati painting is static and animation is dynamic, the importance of picture composition is very important to both of them. It can be said that poetry, books, paintings and prints are more like flying into the picture, allowing literati painters to rethink the layout of their composition. If the position is right, there will be a new sense of picture. If the poem or print in the literati painting is moved to another position, it will probably express another state of mind. From this, we can imagine that an object in an animated picture is more like a poem, a book, a print in the picture, a change in the position of the picture will make a change in the composition of the picture. Animation should take into account the picture layout meaning before and after motion, without staring at static split-mirror composition. In the animated short film Someone's Eyes (Fig 6), at first the little girl is playing on the left side of the picture, and the composition is unbalanced, which makes people feel the loneliness of the little girl. At this moment, a kitten was painted in the picture (Fig 7), whichever makes us feel that the loneliness of the little girl will be much less. After that, the plaything is connected with the happy play of the little girl and the cat. With the growth of the little girl, the picture of the balance is broken again, and the little girl is out of the picture, and the picture is weightless again, leaving the lonely cat.

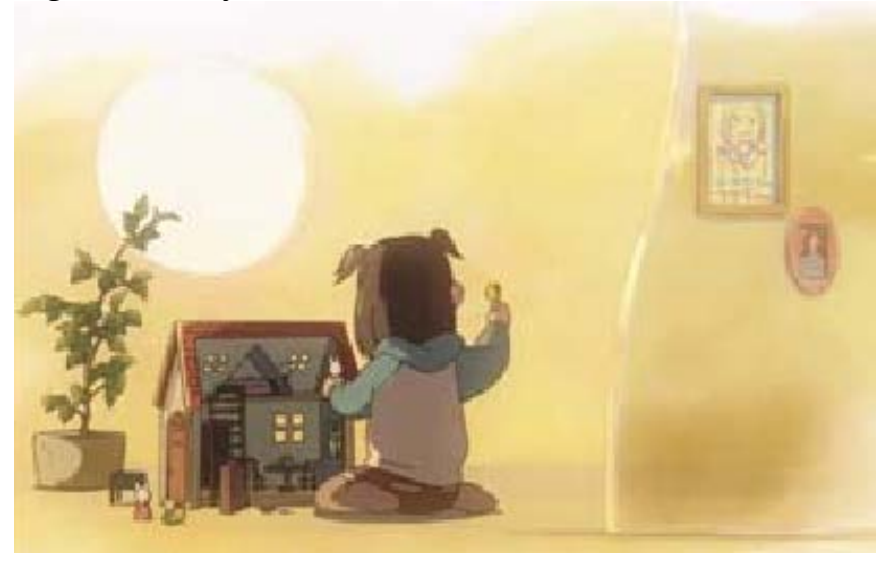

Fig 6. Screenshot in Someone's Eyes. 


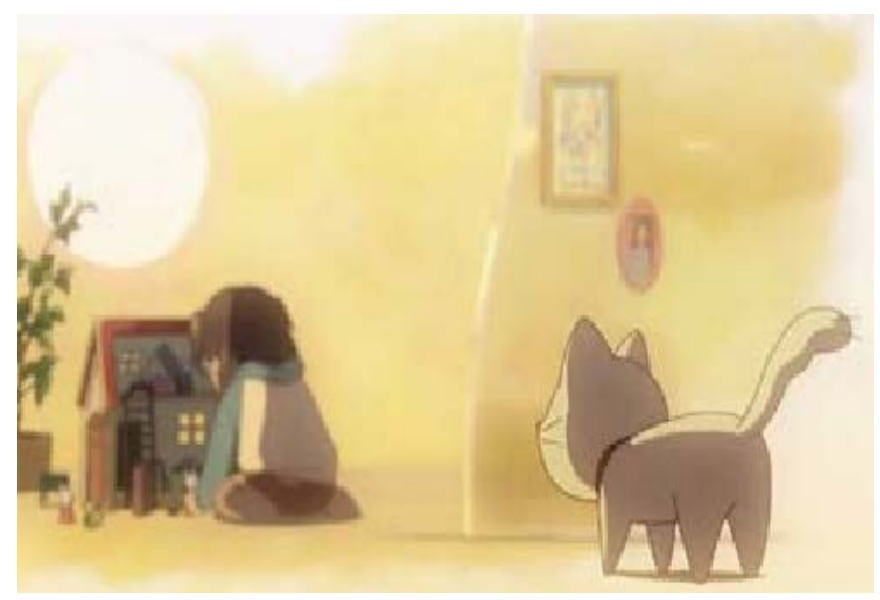

Fig 7. Screenshot in Someone's Eyes.

\section{References}

[1] Yu Na. Application of Ink Painting Elements in Modern Multidimensional Animation [J]. 2017, (19): 12 -129.

[2] Ge Zongnan. Cold thinking on the Great Leap forward of Chinese Animation Education [J]. Journal of Jilin University of Art, 2008. (01): 51-55.

[3] Lai Liangxin. Design and implementation of 3D Ink Animation [J]. Art Education, 2015, (08): 62-63.

[4] Pang Like. The Application of National Elements in the Creation of Animated Original Paintings [J]. The world of literature, 2012, (22): 60+62.

[5] Luo Songyi. The Application of Chinese figure painting in Animated Film [J]. Movie Literature, 2011, (19): 65-66. 